\title{
Curvature-based Human Body Parts Segmentation in Physiotherapy
}

\author{
Francis Deboeverie ${ }^{1}$, Roeland De Geest ${ }^{2}$, Tinne Tuytelaars ${ }^{2}$, Peter Veelaert ${ }^{1}$ and Wilfried Philips ${ }^{1}$ \\ ${ }^{1}$ Department of Telecommunications and Information Processing, Image Processing and Interpretation, UGent/iMinds, \\ St-Pietersnieuwstraat 41, 9000 Ghent, Belgium. \\ ${ }^{2}$ ESAT-PSI, Processing Speech and Images, KU Leuven/iMinds, \\ Kasteelpark Arenberg 10, 3001 Leuven, Belgium. \\ \{Francis.Deboeverie, Peter.Veelaert, Wilfried.Philips\}@telin.ugent.be, \\ $\{$ Roeland.DeGeest, Tinne.Tuytelaars\}@esat.kuleuven.be
}

\begin{abstract}
Keywords: $\quad$ Image Segmentation, Human Body Parts Segmentation, Human Body Skeleton Reconstruction
Abstract: Analysing human sports activity in computer vision requires reliable segmentation of the human body into meaningful parts, such as arms, torso and legs. Therefore, we present a novel strategy for human body segmentation. Firstly, greyscale images of human bodies are divided into smooth intensity patches with an adaptive region growing algorithm based on low-degree polynomial fitting. Then, the key idea in this paper is that human body parts are approximated by nearly cylindrical surfaces, of which the axes of minimum curvature accurately reconstruct the human body skeleton. Next, human body segmentation is qualitatively evaluated with a line segment distance between reconstructed human body skeletons and ground truth skeletons. When compared with human body parts segmentations based on mean shift, normalized cuts and watersheds, the proposed method achieves more accurate segmentations and better reconstructions of human body skeletons.
\end{abstract}

\section{Introduction}

Human body segmentation divides images of human bodies into segments that coincide as well as possible with meaningful parts of the human body, like torso, arms or legs. Segmenting human body images is a first step to computer vision-based analysis of human movements patterns (Poppe, 2007; Aggarwal and Ryoo, 2011; Guo and Lai, 2014). Furthermore, it facilitates many useful applications such as surveillance, human action understanding, pose classification, etc. (Juang et al., 2009; Liang et al., 2009; Hou and Pang, 2011; Xiao et al., 2014). Reliable segmentation leads to good qualitative movement analysis, for instance in physiotherapy. Movement analysis helps athletes, for instance gymnasts, to improve their performance and to reduce the risk of injury (Bartlett, 2007). Analysis of human movements is often performed by finding human body configurations in human body skeleton reconstructions (Mori et al., 2004). However, in order to accurately reconstruct these human body skeletons, human body parts segmentation is still challenging.

In this work, greyscale images of human bodies are segmented into smooth surface segments (intensity patches) and then approximated with maps of polynomial surfaces. The main novelty in this paper is that we show that human body parts are approximated by nearly cylindrical surfaces, of which the axes of minimum curvature accurately reconstruct the human body skeleton. For the reconstructed human body skeleton, the branches generally coincide with the real human body bones, because the cylindrical surfaces have the same shape as the limbs.

To find surface segments that are folded in a certain way, we use a curvature-based segmentation algorithm (Deboeverie et al., 2013) that divides greyscale images with adaptive region growing based on low-degree polynomial fitting. Adaptive refers to the use of a local neighbourhood to add pixels, while adapting the shape (or degree) of the function is based on global behaviour. In this sense there is some local flexibility, while the global behaviour is determined by a more straightforward characterization, such as being concave or convex. The curvature point of view approach is based on the property that, because of Lambert's cosine law (Lambert, 1760), when the light comes mainly from one direction, the intensity surface of an image has the same shape as the object surface itself. For instance, human skin is such a diffusely reflecting surface. Then, a human body, which resembles a convex sphere with small concavities, will be seen as a collection of intensity patches of concave functions, and smaller patches of convex 
functions (Wagemans et al., 2010).

We extensively evaluate human body segmentation on images of an athlete performing different exercises. As a measure for segmentation quality, we examine a line segment distance (Gao and Leung, 2002) between human body skeleton reconstructions and ground truth skeletons. Considering this quality measure, we find that the proposed segmentation outperforms human body segmentation techniques based on mean shift (Comaniciu and Meer, 2002; Porikli and Tuzel, 2003), segmentations based on normalized cuts (Shi and Malik, 2000; Li et al., 2011) and segmentations based on marker-controlled watersheds (Vincent and Soille, 1991; Park et al., 1999).

Many methods related to the segmentation of a human body into its different parts have been proposed by researchers. The most recent papers consider human body segmentation in videos (Juang et al., 2009; Hsieh et al., 2010; Liu et al., 2011; Shao et al., 2012) as well as in static images (Srinivasan and Shi, 2007; Barnard and Heikkila, 2008; Li et al., 2011; Li et al., 2014). For videos, the methods are performed on human bodies which are segmented by using a background model and motion information, which make them infeasible for static images. The methods for static images are further classified into matchingbased (Mori and Malik, 2006), part-based (Mori et al., 2004; Hu et al., 2006) and model-based (Lee and Cohen, 2006; Hu et al., 2009) methods. Matching-based methods compare human body features (such as shape contexts (Mori and Malik, 2006)) in a test image with those in a large set of labelled images. Part-based approaches detect the candidates of each body part and construct the best assembly according to some predefined human body configuration constraints. Modelbased methods firstly generate a large number of hypotheses of human body configurations and then recover the human body configuration by minimizing the errors between the hypotheses and the image. Different from the methods in literature, the approach in this work does not require a training test scheme or a model hypothesis on the human body. Furthermore, none of these techniques consider a segment curvature approach for segmentation. As we will show in the results, considering the curvature in the segmentation process enhances the reconstruction of human body skeletons.

The remainder of this paper is organized as follows. Section 2 describes the human body segmentation algorithm, which is an adaptive region growing algorithm based on constructive polynomial surface fitting. Section 3 describes how to classify human body segments based on the polynomial curvature. Section 4 presents the results of human body segmentation and classification. Finally, we conclude our paper in Section 5.

\section{Human body parts segmentation}

Greyscale human body images are segmented with curvature-based segmentation. Curvature-based segmentation finds surface segments that are folded in a certain way. Therefore, we study an adaptive fitting technique for representing sets of image pixels with geometric primitives. A geometric primitive is a polynomial function describing the variation of grey values in a region. The problem we study is that of finding a region of maximal size in which grey values can be well approximated by a polynomial function. We also consider the related problem of finding the best degree of the polynomial function.

To achieve above-mentioned purposes, we use an adaptive region growing algorithm (Deboeverie et al., 2013) based on constructive polynomial fitting (Veelaert, 1997; Veelaert and Teelen, 2006). Region growing is the process of examining neighbouring pixels of initial seed pixels and determining whether the neighboring pixels should be added to the region. Polynomial fitting fits geometric primitives to pixels. This primitive extraction algorithm determines subsets of pixels that lie on a geometric primitive or close to it. How well a subset corresponds to a primitive is quantified by an $L_{\infty}$ fitting cost (approximation error).

The algorithm finds regions of maximal size in which a low-degree polynomial approximation using a specified $L_{\infty}$ fitting cost criterion exists. Let $f\left(x_{i}, y_{i}\right)$ represent the image intensities. Let $G$ be a vector space of fitting functions, for instance, the vector space of bivariate polynomial functions of total degree $d$ :

$$
g(x, y)=\sum_{k=0}^{d} \sum_{l=0}^{k} \alpha_{l, k-l} x^{l} y^{k-l},
$$

where each polynomial is characterized by $n=(d+$ 1) $(d+2) / 2$ coefficients $\alpha_{l, k-l}$.

The accuracy of fitting $g(x, y)$ over the segment $S$ is measured with the $L_{\infty}$ fitting cost. This fitting cost is defined as

$$
r(S ; g)=\max _{(x, y) \in S}|g(x, y)-f(x, y)| .
$$

The best fit is the polynomial function $g(x, y)$ in $G$ for which $r(S ; g)$ is minimal. This minimal cost is denoted as $r(S)$, i.e,

$$
r(S)=\min _{g \in G} r(S ; g) .
$$

$L_{\infty}$ fitting costs are computed very efficiently by constructive fitting (Veelaert, 1997; Veelaert and Teelen, 2006). The emphasis of constructive fitting is on 


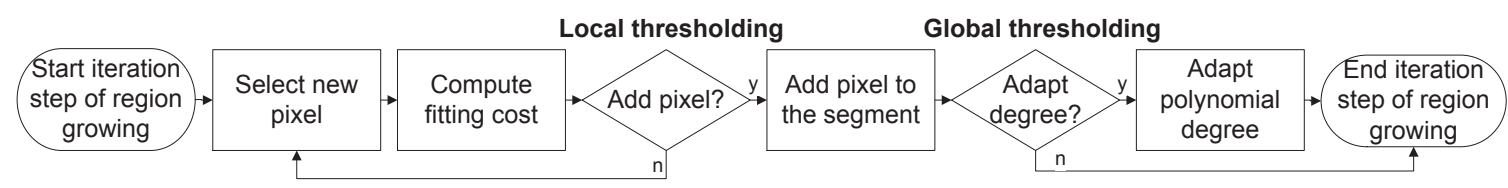

Figure 1: Flow chart of one iteration step in our adaptive region growing. Two key phases are distinguished: the first determines if a new pixel is to be added to a segment, while the second phase controls if the polynomial degree is adapted.

the calculation and estimation of the fitting cost from elemental subsets. Elemental subsets are the smallest subsets that have a nontrivial fitting cost. Estimating the fitting cost with elemental subsets boils down to a sampling of the region. Sampling in this context refers to measuring the fitting cost in only a few pixels of the region and then estimating the fitting cost of the entire region based on these measurements. The selection of elemental subsets in a region can be performed locally as well as globally. A local elemental subset is an elemental subset which has been selected from the pixels in a small part of the region. Likewise, a global elemental subset is an elemental subset which has been selected from the pixels in the entire region. From local and global elemental subsets we compute so-called local and global fitting costs, respectively.

Local and global fitting costs are combined with a strategy for image segmentation in a region growing process with adaptive thresholding (Deboeverie et al., 2013). Adaptive refers to the use of a local neighbourhood to add pixels, while adapting the shape (or degree) of the function is based on global behaviour. In this sense there is some local flexibility, while the global behaviour is determined by a more straightforward characterization, such as being concave or convex. One iteration step of adaptive region growing is visualised in the flowchart in Figure 1. Two key phases are distinguished: the first determines if a new pixel is to be added to a segment, while the second phase controls if the polynomial degree is adapted. Thresholding of the local behaviour $X_{k}$ of the fitting costs controls if a new pixel $p_{k}$ is added to the segment $S_{k-1}=\left\{p_{0}, p_{1}, \ldots, p_{k-1}\right\}$. This is when $X_{k}$ is lower than the threshold $T_{X}$. When $X_{k}$ exceeds $T_{X}$, i.e. when adding $p_{k}$ would increase the fitting cost significantly more than on average, $p_{k}$ is not added to $S_{k-1}$. Thresholding of the global behaviour $Y_{k}$ of the fitting costs controls if the polynomial degree is adapted. The polynomial degree is increased by one, when the global variation of grey values is becoming too large. This is when $Y_{k}$ exceeds the threshold $T_{Y}$. Then, $Y_{k}$ is recomputed for this new degree. In fact, the degree increases until either $Y_{k}$ is again within the limit of the threshold $T_{Y}$, or a maximum degree is exceeded. A maximal polynomial degree of two is sufficient to expand the segment along smooth flat, planar, convex, concave and saddle intensity functions.

From the segmentation it is known that each surface segment can be approximated by a low-degree polynomial surface as in Eq. 1. Then, the intensity function of a segment is represented by its polynomial coefficients. The coefficients are computed after all segments have been found. A method for computing the best $L_{\infty}$ fit is described in (Veelaert, 2012).

\section{Human body parts classification}

This section describes how to classify human body segments based on the curvatures of the approximating polynomial surfaces, in order to reconstruct the human body skeleton.

Convex, concave or saddle like behaviour of a second-degree polynomial surface $g(x, y)$ as in Eq. (1) is defined by the signs of the eigenvalues of the Hessian matrix:

$$
H(g)=\left[\begin{array}{ll}
\frac{\partial^{2} g}{\partial^{2} x} & \frac{\partial^{2} g}{\partial x \partial y} \\
\frac{\partial^{2} g}{\partial x \partial y} & \frac{\partial^{2} g}{\partial^{2} y}
\end{array}\right] .
$$

The entries of the matrix $H(g)$ are the second order derivatives of the surface with respect to $x$ and $y$ coordinates. For a quadratic surface, the second derivatives are constant and hence $H(g)$ is independent of the location of the pixel in the segment. From Eq. (1), we find

$$
H(g)=\left[\begin{array}{cc}
2 \alpha_{2,0} & \alpha_{1,1} \\
\alpha_{1,1} & 2 \alpha_{0,2}
\end{array}\right] .
$$

The maximum and minimum curvatures are determined by the eigenvalues of this matrix, which are found by solving the following characteristic equation:

$$
H k=\lambda k .
$$

The homogeneous system $(H-\lambda I) k=0$ has a nonzero solution if the determinant of its coefficient matrix is zero:

$$
\left|\begin{array}{cc}
2 \alpha_{2,0}-\lambda & \alpha_{1,1} \\
\alpha_{1,1} & 2 \alpha_{0,2}-\lambda
\end{array}\right|=0 .
$$

The matrix $\mathrm{H}$ is symmetric, hence the solution yields two real values $\lambda_{1}$ and $\lambda_{2}$. Both $\lambda_{1}$ and $\lambda_{2}$ are positive for a convex surface and negative for a concave 
surface. Eigenvalues have opposite signs for a saddle surface. One of the eigenvalues is zero for a cylindrical surface.

The product of the two eigenvalues gives the Gaussian curvature $G=\lambda_{1} \lambda_{2}$. The Gaussian curvature is an intrinsic measure of curvature, i.e., its value depends only on how distances are measured on the surface, not on the way it is isometrically embedded in space. The Gaussian curvature is zero when one of the eigenvalues is zero, which corresponds to a cylindrical surface, as when both are zero, which indicates a plane.

The eigenvectors from the characteristic Eq. (7) point to the direction of maximum and minimum curvatures. The azimuth of maximum curvature $\theta$ is given by

$$
\theta=\arctan \left(\frac{k_{11}}{k_{12}}\right),
$$

where $k_{11}$ and $k_{12}$ are components of the eigenvector corresponding to the largest eigenvalue. The direction of minimum curvature is orthogonal to the direction of maximum curvature. In the results, we show that the axes of minimum curvature reconstruct the human body skeleton.

\section{Results}

Experimental video data includes an athlete performing 10 different exercises. Figure $2(a)$ shows a greyscale human body image of the athlete with image size $130 x 180$. As usual in physiotherapy, the athlete only wears short pants. The availability of a diffuse reflecting skin surface makes these images very suitable to test the segmentation method on. Ground truth skeleton data of the human body is obtained from markers which are labelled on the joints of the athlete by physiotherapists. An example of a ground truth skeleton is shown in Figure $2(b)$. This ground truth data is used to evaluate the method for human body segmentation. Figure $2(c)$ shows a first segmentation result of a human body image. The image is segmented into 40 surface segments. The blue, green and red colours in the segmented image correspond to zero, first and second degree polynomial surfaces, respectively. We ascertain that many surface segments correspond to meaningful parts of the human body, such as the arms, the legs and the torso. Figure $2(d)$ shows the best fit polynomial surfaces approximating the surface segments. The result is a reconstructed image of the original image. The human body parts are nicely reconstructed from the lowdegree polynomial surfaces. An example of convex, concave or saddle like behaviour is shown in Figure 2

\begin{tabular}{lc}
\hline Performance statistics & \\
\hline image size & $180 \times 130$ \\
mean fitting cost & $3.97 \pm 0.42$ \\
\#surfaces & $17.83 \pm 3.14$ \\
\hline
\end{tabular}

Table 1: This table always shows the mean and standard deviation of the mean fitting cost and the number of surfaces, respectively. Only very few surface segments are needed to represent a human body image.

(e). The magenta, cyan and yellow colours correspond to convex, concave and saddle like behaviour, respectively. We find concave polynomial surfaces for the arms, the legs and the torso. We experimentally found that human body parts which are shadowed by other human body parts are often approximated by convex polynomial surfaces. Figure $2(f)$ shows the axes of minimum curvature of the polynomial surfaces. These axes go through the center points of the segments. For the human body segments, these axes coincide with the real human body bones. Together, they form a reconstructed human body skeleton.

To find the optimal parameter set of adaptive region growing, we measure the image approximation accuracy with a surface area weighted mean of the $L_{\infty}$ fitting costs of the polynomial surfaces . A high approximation accuracy (low mean fitting cost) leads to a high number of smaller segments, providing a good approximation quality. On the other hand, a low approximation accuracy (high mean fitting cost) leads to a low number of larger segments, providing approximation quality less well. Depending on the desired purpose (approximation or segmentation), one has to find a good balance between the size of the segments and the quality of the approximated images. For the results in this work, we set the segmentation parameters $T_{X}=0.8$ and $T_{Y}=4.8$, preserving a good size of the segments to perform analysis. These parameters have been manually tuned on a small number of images. When considering a set of 200 human body images, Table 1 always shows the mean and standard deviation of the mean fitting cost and the number of surfaces, respectively. We find that adaptive region growing divides a human body image in only very few surface segments.

The graph in Figure 3 plots the numbers of surface segments when segmenting human body images in function of different mean fitting costs (4). We find that for mean fitting costs above 5 , which corresponds to low reconstruction accuracies, the mean numbers of surface segments remains more or less constant. This means that there is a small stable set of large segments. In contrast, for mean fitting costs below 5, which corresponds to high reconstruction accuracies, 

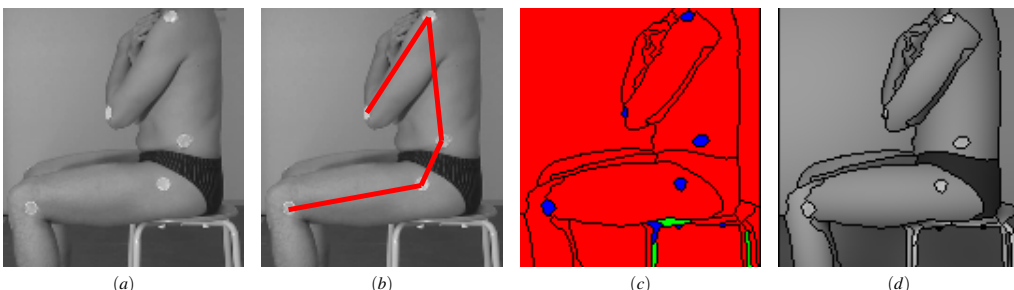
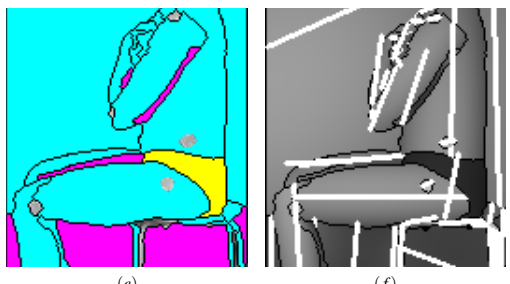

Figure 2: $(a)$ : A greyscale human body image with image size 130x180. $(b)$ : The ground truth human body skeleton. $(c)$ : The human body image is segmented into 40 surface segments. The blue, green and red colours in the segmented image correspond to zero, first and second degree polynomial surfaces, respectively. $(d)$ : The surface reconstructed human body image. $(e)$ : The convex, concave or saddle like behaviour of the polynomial surfaces, indicated by the colours magenta, cyan and yellow, respectively. $(f)$ : The axes of minimum curvature of the nearly cylindrical surfaces approximating the human body parts.

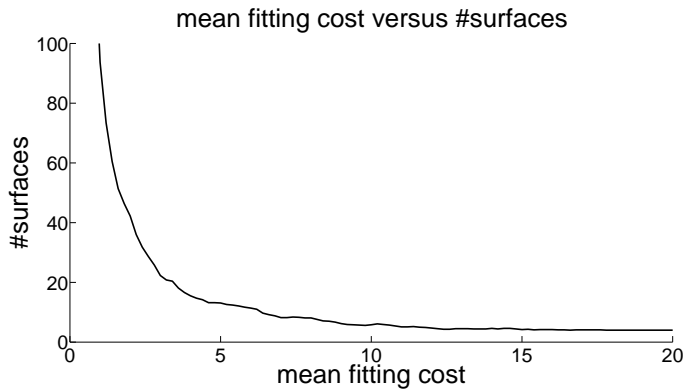

Figure 3: The numbers of surface segments of human body images in function of mean fitting costs.

the mean number of surface segments grows exponentially in function of the mean fitting cost. This means that there are many small segments.

Figure 4 shows the axes of minimum curvature of the second-degree polynomial surfaces approximating the human body parts in Figure $2(e)$. For these surfaces, Table 2 always shows the minimum and maximum curvatures, the Gaussian curvatures (Section 3 ) and the azimuths of minimum curvatures, respectively. From these values, we conclude that the polynomial surfaces approximating human body parts are nearly cylindrical, since the Gaussian curvature is zero when one of the eigenvalues is zero. The corresponding axes of minimum curvature reconstruct the human body skeleton. They coincide with the ground truth human body skeleton in Figure $2(b)$.

In order to qualitatively evaluate human body segmentation, we match the reconstructed human body skeleton to the ground truth skeleton data with a Line Segment Distance (LSD) as proposed in (Gao and Leung, 2002). The LSD is useful in skeleton matching, because it encourages one-to-one mapping of similar lines. Given two sets of line segments $M=$ $\left\{m_{1}, m_{2}, \ldots, m_{p}\right\}$ and $T=\left\{t_{1}, t_{2}, \ldots, t_{q}\right\}$, the distance

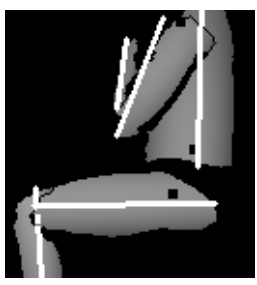

Figure 4: This figure shows the axes of minimum curvature of the nearly cylindrical surfaces approximating the human body parts. When matched to the ground truth skeleton, the LSD is 3.90 .

\begin{tabular}{lcccc}
\hline & $\lambda_{1}$ & $\lambda_{2}$ & $\mathrm{G}$ & $\theta$ \\
\hline lower leg & -0.0063 & -0.3668 & 0.0044 & 81 \\
upper leg & -0.0020 & -0.1750 & 0.0004 & -2 \\
lower arm & -0.0058 & -2.0147 & 0.1907 & -75 \\
upper arm & -0.0150 & -0.1815 & 0.0076 & -53 \\
torso & -0.0054 & -0.1184 & 0.0007 & -87 \\
\hline
\end{tabular}

Table 2: This table always shows the minimum and maximum curvatures, the Gaussian curvatures and the azimuths of minimum curvatures of the second-degree polynomial surfaces approximating the human body parts in Fig. $2(e)$.

between two line segments $m_{i}$ and $t_{j}$ is defined as

$$
d\left(m_{i}, t_{j}\right)=\sqrt{d_{\theta}^{2}\left(m_{i}, t_{j}\right)+d_{\|}^{2}\left(m_{i}, t_{j}\right)+d_{\perp}^{2}\left(m_{i}, t_{j}\right)}
$$

where $d_{\theta}\left(m_{i}, t_{j}\right)$ is the orientation distance, $d_{\|}\left(m_{i}, t_{j}\right)$ is the parallel distance and $d_{\perp}\left(m_{i}, t_{j}\right)$ is the perpendicular distance between $m_{i}$ and $t_{j}$ (Gao and Leung, 2002). The line segments $m_{i}$ and $t_{j}$ form a corresponding pair if $d\left(m_{i}, t_{j}\right)$ is a minimum over all combinations of $t_{j}$. From the pairs of matching line segments, a length weighted matching cost $C(M, T)$ is computed:

$$
C(M, T)=\frac{1}{\sum_{m_{i} \in M} l_{m_{i}}} \sum_{m_{i} \in M} l_{m_{i}} \min _{t_{j} \in T} d\left(m_{i}, t_{j}\right),
$$

where $l_{m_{i}}$ is the length of line segment $m_{i}$. This matching cost is used as a quality measure for human 


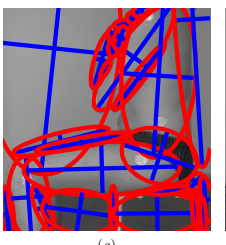

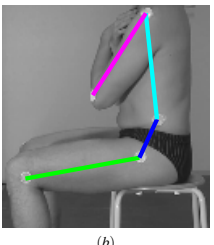

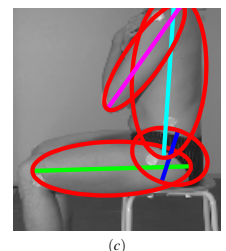

Figure 5: $(a)$ : The human body image segments represented by ellipses and their axes. (b): The ground truth human body skeleton. $(c)$ : The axes of the ellipses that match with the ground truth skeleton. The corresponding line segment pairs are indicated by the same colour. The LSD is 6.34 .

body segmentation, where a lower LSD corresponds to a better segmentation quality. For instance, matching the axes in Figure $2(f)$ to the ground truth skeleton in Figure $2(b)$ results in a LSD of 3.90.

The reconstruction from the axes of minimum curvature of cylinders only applies to the proposed method. In order to compare, we also reconstruct the human body skeleton from the axes of an ellipse model for the surface segments. The reconstruction from the axes of ellipses applies to methods that do not provide curvature information of the surface segments. We obtain ellipses from least-squares fits. An ellipse model for human body parts was earlier proposed in (Park and Aggarwal, 2004; Wu and Aghajan, 2007). An example human body surface segments represented by ellipses and their axes is shown in Figure $5(a)$. To measure the quality of a human body segmentation, we match the axes of the ellipses with the ground truth skeleton using the LSD. Figure $5(c)$ shows the axes of the ellipses that match with the ground truth skeleton in Figure $5(b)$. The corresponding line segment pairs are indicated by the same colour. Here, the LSD is 6.34. The correct axes are matched, they follow almost the same directions as the ground truth skeleton.

Additional results are shown in Figure 6. The columns $(a)$ to $(f)$ show the input images, the segmented images, the surface reconstructed images, the reconstructed human body skeletons from the axes of minimum curvature, the ground truth human body skeletons and the axes of the ellipses representing the segments that match with the ground truth skeletons, respectively. The corresponding line segment pairs are indicated in the same colour. The surface segments of the human bodies are separated from the background by considering the segments close to the markers.

We compare our segmentation based on polynomial surfaces, with human body segmentation algorithms based on mean shift (Comaniciu and Meer, 2002; Porikli and Tuzel, 2003), segmentations based on normalized cuts (Shi and Malik, 2000; Li et al.,

\begin{tabular}{lc}
\hline Line Segment Distance & (lower is better) \\
\hline Polynomial surfaces (cylinders) & $\mathbf{4 . 8 7} \pm \mathbf{1 . 2 1}$ \\
Polynomial surfaces (ellipses) & $6.31 \pm 2.17$ \\
Mean-shift & $10.73 \pm 2.99$ \\
Normalized cuts & $11.64 \pm 3.25$ \\
Marker-controlled watersheds & $16.37 \pm 3.28$ \\
\hline
\end{tabular}

Table 3: This table always shows the means and standard deviations of the LSDs for segmentation with polynomial surfaces, mean shift, normalized cuts and marker-controlled watersheds, respectively. For our method, beside the human body skeleton reconstructions from the axes of cylinders, we also make a variant of our method which produces human body skeleton reconstructions from the axes of ellipses.
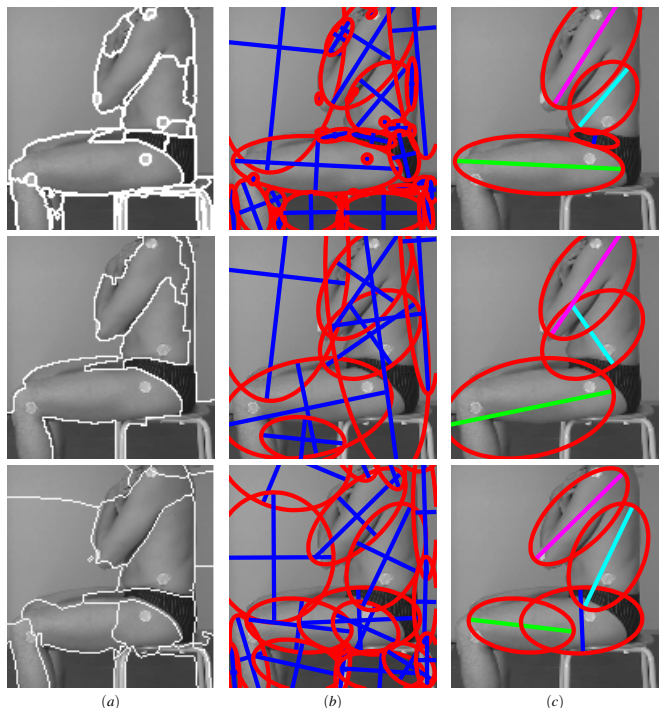

Figure 7: $(a)$ : The segmentation of a human body image with mean shift, normalized cuts and marker-controlled watersheds, respectively. $(b)$ : The segments represented by ellipses and their axes. $(c)$ : The axes of the ellipses that match with the ground truth skeleton. The LSDs are 10.56, 12.14 and 17.61 , respectively.

2011) and segmentations based on marker-controlled watersheds (Vincent and Soille, 1991; Park et al., 1999). Output examples of human body segmentation with mean shift, normalized cuts and markercontrolled watersheds are shown in column $(a)$ in Figure 7. Column $(b)$ in Figure 7 shows the segments represented by ellipses and their axes. Column $(c)$ in Figure 7 shows the axes of the ellipses that match with the ground truth skeleton using the LSD. The LSDs for human body segmentation with mean shift, normalized cuts and watersheds are 10.56, 12.14 and 17.61 , respectively.

Table 3 always shows the mean and standard deviation of the LSDs of human body segmentations based on polynomial surfaces, mean shift, normal- 


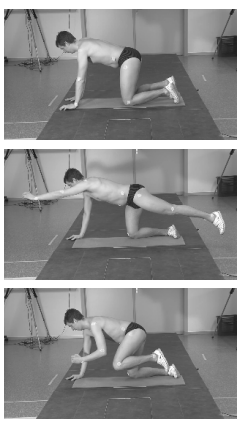

(a)

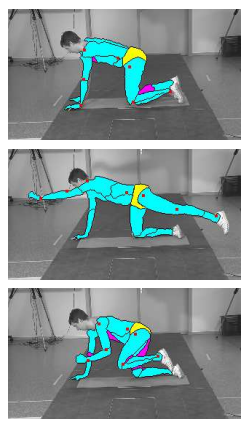

(b)

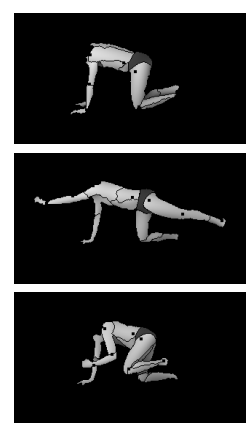

(c)
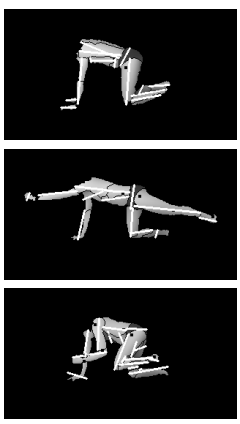

$(d)$

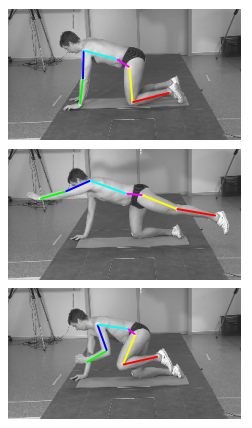

$(e)$

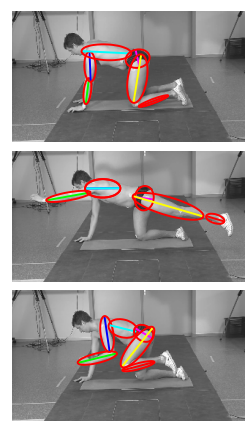

$(f)$

Figure 6: $(a)$ : Input images. $(b)$ : The segmented images. Many surface segments correspond to meaningful parts of the human body, such as the arms, the legs and the torso. The magenta, cyan and yellow colours correspond to convex, concave and saddle like polynomial surfaces. We find concave polynomial surfaces for the arms, the legs and the torso, while shadowed human body parts are often approximated by convex polynomial surfaces. $(c)$ : The surface reconstructed human body images. $(d)$ : Reconstructed human body skeletons from the axes of minimum curvature of the polynomial surfaces approximating the human body. $(e)$ : The ground truth human body skeletons. $(f)$ : The axes of the ellipses representing the segments that match with the ground truth skeletons. The corresponding line segment pairs are indicated by the same colour.

\begin{tabular}{lccccc}
\hline LSD (lower is better) & lower leg & upper leg & lower arm & upper arm & torso \\
\hline Polynomial surfaces (cylinders) & $\mathbf{1 . 9 4}$ & $\mathbf{1 . 8 1}$ & $\mathbf{2 . 2 6}$ & $\mathbf{1 . 1 3}$ & $\mathbf{4 . 1 2}$ \\
Polynomial surfaces (ellipses) & 2.56 & 2.48 & 2.88 & 1.81 & 7.55 \\
Mean-shift & 4.17 & 3.64 & 4.05 & 2.83 & 19.83 \\
Normalized cuts & 4.69 & 3.25 & 4.54 & 2.21 & 27.33 \\
Marker-controlled watersheds & 5.03 & 4.86 & 5.39 & 2.96 & 24.84 \\
\hline
\end{tabular}

Table 4: This table always shows the means of the LSDs for segmentation of individual human body parts with polynomial surfaces, mean shift, normalized cuts and marker-controlled watersheds, respectively.

ized cuts and marker-controlled watersheds, respectively. For our method, beside the human body skeleton reconstructions from the axes of cylinders, we also make a variant of our method which produces human body skeleton reconstructions from the axes of ellipses. We find that the LSDs of other segmentation methods are higher than the LSD of our segmentation. Furthermore, a human body skeleton reconstruction with the axes of cylinders is more accurate than a reconstruction with the axes of ellipses. When we consider the means of the LSDs for segmentation of individual human body parts in Table 4, we find that our method outperforms the other techniques in all cases, especially for the skeleton reconstruction of the torso.

\section{Conclusion}

In this work, we perform human sports activity analysis with computer vision by reliable segmentation of the human body into meaningful parts, such as arms, torso and legs. Human body segmentation is performed on greyscale human body images by adaptive region growing based on constructive poly- nomial fitting. Human body images are represented by flat, planar, convex, concave and saddle polynomial surfaces with a variable fitting error. The lowdegree polynomial surfaces correspond to meaningful human body features. We find that human body parts are often represented as nearly cylindrical surfaces, of which the axes of minimum curvature reconstruct the human body skeleton accurately. The proposed method, which provides human body skeleton reconstructions from curvature information of the surface segments, outperforms existing segmentation techniques that do not provide this information. By sharing the analysis of exercises with the physiotherapist and the athlete, the athlete can improve the way he is performing excercises in order to obtain optimal sports or recovery results.

\section{Acknowledgements}

The work was financially supported by iMinds and IWT through the project 'LittleSister' and by FWO through the project G.0.398.11.N.10 "Multicamera human behavior monitoring and unusual event detection". 


\section{REFERENCES}

Aggarwal, J. K. and Ryoo, M. (2011). Human activity analysis: A review. ACM Computing Surveys, 43(3).

Barnard, M. and Heikkila, J. (2008). Body part segmentation of noisy human silhouette images. In Proc. of IEEE Int. Conf. on Mult. and Expo, pages 1189-1192.

Bartlett, R. (2007). Analysing Human Movements Patterns. Routledge.

Comaniciu, D. and Meer, P. (2002). Mean shift: A robust approach toward feature space analysis. IEEE Trans. on Pattern Analysis and Machine Intelligence, 24(5):603-619.

Deboeverie, F., Veelaert, P., and Philips, W. (2013). Image segmentation with adaptive region growing based on a polynomial surface model. Journal of Electronic Imaging, 22(4).

Gao, Y. and Leung, M. K. (2002). Face recognition using line edge map. IEEE Trans. on Pattern Analysis and Machine Intelligence, 24(6):764-779.

Guo, G. and Lai, A. (2014). A survey on still image based human action recognition. Pattern Recognition, 47(10):3343 - 3361.

Hou, Y.-L. and Pang, G. K. H. (2011). People counting and human detection in a challenging situation. IEEE Trans. on Systems, Man, and Cybernetics - Part A: Systems and Humans, 41(1):24-33.

Hsieh, J.-W., Chuang, C.-H., Chen, S.-Y., Chen, C.-C., and Fan, K.-C. (2010). Segmentation of human body parts using deformable triangulation. IEEE Trans. on Systems, Man, and Cybernetics - Part A: Systems and Humans, 40(3):596-610.

$\mathrm{Hu}, \mathrm{Z}$., Lin, X., and Yan, H. (2006). Torso detection in static images. In Proc. of Int. Conf. on Signal Processing.

$\mathrm{Hu}$, Z., Wang, G., Lin, X., and Yan, H. (2009). Recovery of upper body poses in static images based on joints detection. Pattern Recognition Letters, 30:503-512.

Juang, C.-F., Chang, C.-M., Wu, J.-R., and Lee, D. (2009). Computer vision-based human body segmentation and posture estimation. IEEE Trans. on Systems, Man, and Cybernetics - Part A: Systems and Humans, 39(1):119-133.

Lambert, J. (1760). Photometria Sive de Mensura de Gratibus Luminis. Colorum et Umbrae. Augsberg, Germany: Eberhard Klett.

Lee, M. W. and Cohen, I. (2006). A model-based approach for estimating human $3 \mathrm{~d}$ poses in static images. IEEE Trans. on Pattern Analysis and Machine Intelligence, 28(6):905-916.

Li, S., Lu, H.-C., Ruan, X., and Chen, Y.-W. (2011). Human body segmentation based on deformable models and two-scale superpixel. Pattern Analysis and Applications, pages $1-15$.

Li, S., Shao, X., et al. (2014). Human body segmentation via data-driven graph cut. IEEE Trans. on Cybernetics. DOI:10.1109/TCYB.2014.2301193.

Liang, Y.-M., Shih, S.-W., Shih, C.-C., Liao, H.-Y. M., and Lin, C.-C. (2009). Learning atomic human actions using variable-length markov models. IEEE Trans. on
Systems, Man, and Cybernetics - Part B: Cybernetics, 39(1):268-280.

Liu, Q., Li, H., and Ngan, K. N. (2011). Automatic body segmentation with graph cut and self-adaptive initialization level set (sails). J. Vis. Commun. Image R., 22:367-377.

Mori, G. and Malik, J. (2006). Recovering 3d human body configurations using shape contexts. IEEE Trans. Pattern Analysis Machine Intelligence, 28(7):1052-1062.

Mori, G., Ren, X., Efros, A. A., and Malik, J. (2004). Recovering human body configuration: Combining segmentation and recognition. In Proc. of Computer Vision and Pattern Recognition, pages 326-333.

Park, J.-S., Oh, H.-S., Chang, D.-H., and Lee, E.-T. (1999). Human posture recognition using curve segments for image retrieval. In Proc. of SPIE.

Park, S. and Aggarwal, J. K. (2004). Semantic-level understanding of human actions and interactions using event hierarchy. In Proc. of Computer Vision and Pattern Recognition Workshop, page 12.

Poppe, R. (2007). Vision-based human motion analysis: An overview. Computer vision and image understanding, 108(1):4-18.

Porikli, F. and Tuzel, O. (2003). Human bodytracking byadaptive background models and mean-shift analysis. In IEEE Int. Workshop on Performance Evaluation of Tracking and Surveillance.

Shao, L., Ji, L., Liu, Y., and Zhang, J. (2012). Human action segmentation and recognition via motion and shape analysis. Pattern Recognition Letters, 33:438-445.

Shi, J. and Malik, J. (2000). Normalized cuts and image segmentation. IEEE Trans. on Pattern Analysis and Machine Intelligence, 22(8):888-905.

Srinivasan, P. and Shi, J. (2007). Bottom-up recognition and parsing of the human body. In Proc. of Computer Vision and Pattern Recognition, pages 1-8.

Veelaert, P. (1997). Constructive fitting and extraction of geometric primitives. CVGIP: Graphical Models and Image Processing, 59(4):233-251.

Veelaert, P. (2012). Separability and tight enclosure of point sets, digital geometry algorithms. Theoretical Foundations and Appl. to Computational Imaging, 2.

Veelaert, P. and Teelen, K. (2006). Fast polynomial segmentation of digitized curves. In Proc. of Discrete Geometry for Computer Imagery, pages 482-493.

Vincent, L. and Soille, P. (1991). Watersheds in digital spaces: An efficient algorithm based on immersion simulations. IEEE Trans. on Pattern Analysis and Machine Intelligence, 13(6):583-598.

Wagemans, J., Doorn, A. J. V., and Koenderink, J. (2010). The shading cue in context. i-Perception, 1(3):159178

Wu, C. and Aghajan, H. (2007). Model-based image segmentation for multi-view human gesture analysis. In Proc. of Advanced Concepts for Intelligent Vision Systems, pages 310-321.

Xiao, Y., Lu, H., and Sun, C. (2014). Pose estimation based on pose cluster and candidates recombination. IEEE Trans. on Circuits and Systems for Video Technology. DOI:10.1109/TCSVT.2014.2347511. 